\title{
Channel-Sharing Strategies in Two-Tier Cellular PCS Systems
}

\author{
Kuo-Jen Lin*, Yu-Chee Tseng**, and Jang-Ping Sheu* \\ * Department of Computer Science and Information Engineering \\ National Central University Chung-Li, 320, Taiwan \\ golden@axp1.csie.ncu.edu.tw \\ sheujp@csie.ncu.edu.tw \\ ** Department of Computer Science and Information Engineering \\ National Chiao-Tung University Hsin-Chu, 300, Taiwan \\ yctseng@csie.nctu.edu.tw
}

\begin{abstract}
A two-tier cellular network is characterized by overlapping of macrocells and microcells in the service area. This overlapping property provides an advantage that traffic loads can be shared by the two tiers to increase the performance of the system. In this paper, we propose two channel-sharing strategies, namely vertical loadsharing and horizontal load-sharing, to better utilize channels of the network. The call loss probability of new calls and call dropping probability of handoffs are developed through analysis and simulation. The results justify the advantage of our strategies over existing strategies.
\end{abstract}

Keywords: cellular network, channel management, load balance, personal communication system, two-tier cellular system.

\section{Introduction}

The Personal Communication System (PCS) is one of the fastest growing industries recently. One inherent limitation to wireless communication is the scarce wireless bandwidth, which can never catch up with the increase of user demand. One way to release the stress is to use a twotier cellular structure to increase channel reuse (or frequency reuse). Resident on the top layer are larger cells called macrocells, while resident on the bottom layer are smaller cells called microcells. Macrocells and microcells can overlap with each other in the service area. Such an arrangement is more dynamic than a single-tier system, and thus can offer a chance to optimize the performance of the system based on factors such as roaming speed of users, level of cloudiness of an area, location management, channel management, etc. Many works have been directed toward this direction [1, 2, 3, 4, 5, 6, 7]. In [1, 2, 3, 7], subscribers are assigned to microcell or macrocell based on their mobility. In [5], calls are classified into several categories depending on their velocities; different handoff thresholds are used for them. It was proposed in [4] to direct call termination and paging on the same tier to reduce paging cost. The velocity threshold to choose tiers is dynamically selected in [6].
The main purpose of this paper is to investigate the possibility of sharing channels between the two tiers. This is very natural because microcells and macrocells will often overlap with each other. The potential advantage is higher channel utilization. A number of works have addressed this issue. In [8,7], a new/handoff call will be directed to the appropriate tier based on its previous speed. However, when there are no available channels on the preferred tier, the call will be directed to the other (un-preferred) tier. This is called an overflow. In [9], only overflow from the low tier to the high tier is allowed, while in [10] overflow from the low tier to the high tier is restricted to only handoffs. In [11], mobile subscribers traveling on the low tier may borrow channels from a pool of reserved handoff channels provided by the high tier.

In [12], two-way overflows between both tiers are considered. Also, a take-back scheme is introduced so as to redirect a call from an un-preferred tier to a preferred tier at the occasions of handoffs. That is, whenever possible, a fast subscriber overflowed to a microcell will be taken back to a macrocell when it crosses any microcell boundary, and vice versa for a slow subscriber. Observing that two continuous cells usually have some overlapping on their radio coverage areas, reference [13] proposes a channel rearrangement scheme by forcing a handset in the overlapping area to take an early handoff prematurely, if the signal quality in the next cell is acceptable. This will vacate a channel in the previous cell. A chaining effect may even take place if this causes a sequence of subscribers to take early handoffs.

The above reviews show the flexibility of two-tier systems in transferring the load to overlapping and neighboring cells. In this paper, we propose a new strategy called vertical channel-sharing in a two-tier system. Suppose that a macrocell is overlapping with $n$ microcells. When a channel request arrives at the macrocell, if the macrocell has no free channels, we can either overflow this call to its corresponding microcell, or force one of the calls on the macrocell to one of the other $n-1$ microcells to vacate a channel. On the contrary, when a channel request arrives at a 


\begin{tabular}{|c|c|c|}
\hline methods & strategies & ways to redirect \\
\hline 8,7 & handoff & 0 \\
\hline 10,9 & overflow (1-way) & 1 \\
\hline 11 & borrow & 1 \\
\hline 12 & overflow (2-way) & 1 \\
\hline 13 & overflow+rearrange & $1+v$ \\
\hline Ours & VHCS & $n+v$ \\
\hline
\end{tabular}

Table 1: Comparison of channel-sharing strategies, where the "VHCS" is the vertical and horizontal channel-sharing

microcell, if the microcell has no free channels, we can either overflow this call to the macrocell, or force one of the calls on the macrocell to one of the other $n-1$ microcells to vacate a channel. Then the call can be overflowed to the macrocell. We observe that such channel-sharing provides a lot more varieties to shift the load among the cells on the two tiers than simply doing overflow. Further supposing that a macrocell is neighboring to $v$ macrocells, we also consider the possibility of taking a horizontal channelsharing to the $v$ neighbor macrocells similar to that in [13]. In our approach, we will take vertical channel-sharing prior to horizontal channel-sharing.

A summary on the numbers of ways to shift a channel request by other schemes and ours is in Table 1. This shows the flexibility of our strategies. Formal analyses are provided to evaluate the performance of our vertical and horizontal channel-sharing. Simulation results are also provided to verify our analyses. The results do justify the benefits of using our strategies.

The rest of this paper is organized as follows. Our channel-sharing strategies are described in Section 2. Call loss probability of new calls is derived in Section 3. Comparisons, including numerical and simulation results, are presented in Section 4. Section 5 concludes this paper.

\section{Vertical and Horizontal Channel-Sharing Strategies}

In the following discussion, we will consider a macrocell $M$, which overlaps with $n$ microcells $m_{1}, m_{2}, \ldots, m_{n}$, and neighbors with $v$ macrocells $M_{1}, M_{2}, \ldots, M_{v}$. Suppose there is a channel request arriving at the macrocell $M$ or one of $v$ microcells $m_{1}, m_{2}, \ldots, m_{v}$. If there is no channel in the cell to satisfy this request, our vertical channelsharing will take place first, trying to vacate a channel by readjusting calls between the two tiers. If this fails, our horizontal channel-sharing will further take place, trying to vacate a channel by shifting calls to the neighboring macrocells $M_{1}, M_{2}, \ldots, M_{v}$. These strategies are detailed in the following.

\subsection{Channel Sharing in the Vertical Direc- tion}

By "vertical", we mean transferring calls between the two tiers. In the following, we separate our discussion into calls arriving at the low tier and the high tier. When there is a channel request to microcell $m_{i}, 1 \leq i \leq n$, the following steps will be executed:

V1. If there is a free channel in $m_{i}$, assign this channel to the request.

V2. Otherwise, "overflow" the request to the macrocell $M$ if there is a free channel in $M$.

V3. Otherwise, pick any call in $M$ such that the call's corresponding microcell, say $m_{j}$, has a free channel. Transfer the call to $m_{j}$ to vacate a channel in $M$, and then "overflow" the channel request to $M$.

For example, in Fig. 1(a), a slow subscriber $A$ arrives at microcell $m_{1}$, which has no free channel. Then $A$ will be overflowed to macrocell $M$ by V2. Since $M$ is full too, the strategy will try to identify a user, say $B$, which can be handoff to microcell $m_{4}$, which has a free channel. Then the channel released by $B$ in the high tier can be used by A.

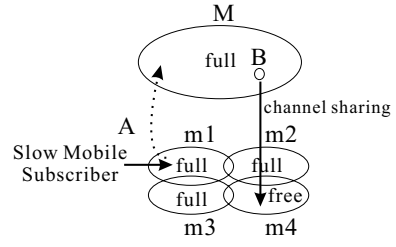

(a)

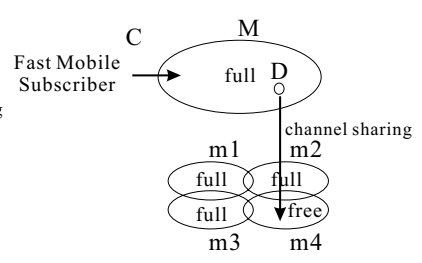

(b)
Fig. 1: Examples of vertical channel-sharing: (a) slow subscriber and (b) fast subscriber.

When there is a channel request to $M$, the following steps will be executed:

V1'. If there is a free channel in $M$, assign this channel to the request.

V2'. Otherwise, "overflow" this request to its corresponding microcell if the microcess has a free channel.

V3'. Otherwise, pick any call in $M$ such that the call's corresponding microcell, say $m_{j}$, has a free channel. Transfer the call to $m_{j}$ to vacate a channel in $M$, and then assign the vacated channel to the request.

For example, in Fig. 1(b), a fast subscriber $C$ moves into macrocell $M$, which has no free channel. Then step V2' will first try to overflow $C$ to its corresponding microcell $m_{1}$. Since $m_{1}$ is full too, step V3' will try to locate a subscriber, say $D$, which can be handoff to $m_{4}$. Then the channel of $D$ can be given to $C$.

\subsection{Channel Sharing in the Horizontal Di- rection}

If the above vertical channel-sharing fails, a horizontal sharing will be taken place. This is done by forcing a subscriber on $M$ take an early handoff as follows. 
H1. Pick any macrocell $M_{i}, 1 \leq i \leq v$, which is neighboring to $M$ such that $M_{i}$ has at least one free channel and there is a subscriber, say $x$, resident in the area that is covered by both $M$ and $M_{i}$.

H2. If H1 succeeds, enforce subscriber $x$ to take an early handoff to $M_{i}$ to vacate a channel. If the channel request is made on the high tier, assign the vacated channel to the request directly; otherwise, overflow the request from the low tier to the high tier to use the vacated channel.

Fig. 2 shows two examples by forcing users $B$ and $D$ on $M$ to take an early handoff to vacate a channel for the requests made by users $A$ and $C$, respectively.

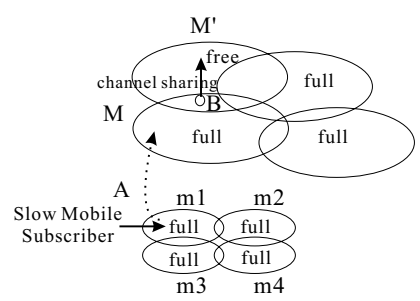

(a)

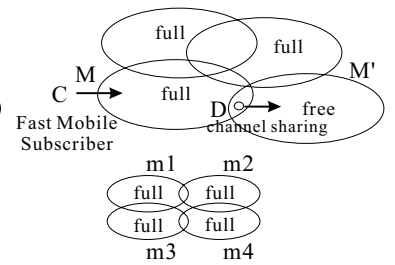

(b)
Fig. 2: Examples of horizontal channel-sharing: (a) slow subscriber and (b) fast subscriber.

We comment that our horizontal channel-sharing happens on the high tier only. Although theoretically it is possible to take horizontal channel-sharing among microcells, we tend to not do so because it is less practical considering the size of microcells. Also, the failure of our vertical channel-sharing implies that there are no free channels in microcells $m_{1}, m_{2}, \ldots, m_{n}$ covered by macrocell $M$. Thus the success possibility of doing so could be quite low.

\section{Performance Analysis on Both Vertical and Horizontal Channel-Sharing}

In this section, we discuss some basic assumptions in our analysis. We assume that each macrocell covers $n$ microcells. We assume that all cells in the same tier are statistically identical, and thus we can focus on the behavior of only one cell and its interaction with neighboring cells. Each macrocell and microcell is assumed to cover a circle. The radius of the circle for a subscriber to take a normal handoff on a macrocell is $r_{n}^{M}$, and that on a microcell $r_{n}^{m}$. However, since there will be some overlapping between two macrocells/microcells, the radius of the circle for a subscriber to take an early handoff on a macrocell is $r_{e}^{M}$, and that on a microcell $r_{e}^{m}$. The early handoff area is between the $r_{e}^{M}$ and $r_{n}^{M}$ for macrocell, and between the $r_{e}^{m}$ and $r_{n}^{m}$ for microcell. By using both vertical and horizontal channel-sharing, a mobile subscriber, when seeing no free channel on its local cell, can take a vertical channel-sharing first. If this fails, a horizontal channel-sharing can be taken. Again, our goal is to derive the call loss probabilities $P_{l f}$ and $P_{l s}$ of new calls for fast and slow subscribers, respectively. As shown in Fig. 3, we have

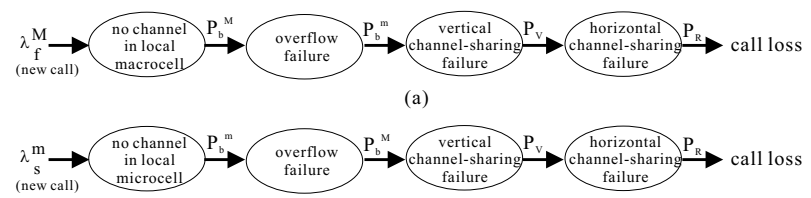

(b)

Fig. 3: Procedures to choose a channel based-on vertical channel sharing and horizontal channel-sharing when a request for a channel arrives: (a) fast subscriber, and (b) slow subscriber.

$$
\begin{aligned}
& P_{l f}=P_{b}^{M} P_{b}^{m} P_{v} P_{R} \\
& P_{l s}=P_{b}^{m} P_{b}^{M} P_{v} P_{R}
\end{aligned}
$$

where $P_{v}$ is the failure probability of vertical channelshaing,

$$
P_{v}=1-P_{s v}\left(1-P_{b}^{m}\right),
$$

where $P_{s v}$ is the probability that a subscriber in macrocell can be rearranged to a microcell:

$$
P_{s v}=1-\left(\frac{n-1}{n}\right)^{c^{M}} .
$$

The $P_{R}$ is the failure probability of horizontal channelsharing,

$$
P_{R}=1-P_{c a n}^{M}\left(1-P_{b}^{M}\right),
$$

where $P_{c a n}^{M}$ is the probability for at least one subscriber staying in early handoff area,

$$
P_{c a n}^{M}=1-\left(\frac{\left(r_{e}^{M}\right)^{2}}{\left(r_{n}^{M}\right)^{2}}\right)^{c^{M}} .
$$

The number of channels for macrocell and microcell are $c^{M}$ and $c^{m}$, respectively. The $P_{b}^{M}$ (resp., $P_{b}^{m}$ ) is the probability that a mobile subscriber sees no free channel in a macrocell (resp., microcell). We can use the Erlang Loss Formula to derive $P_{b}^{M}$ and $P_{b}^{m}$ :

$$
\begin{gathered}
P_{b}^{M}=\frac{\frac{\left(\frac{\lambda_{t f}^{M}}{\mu_{f}^{M}}+\frac{\lambda_{t s}^{M}}{\mu_{s}^{M}}\right)^{c^{M}}}{c^{M !}}}{\sum_{l=0}^{c^{M}} \frac{\left(\frac{\lambda_{t f}^{M}}{\mu_{f}^{M}}+\frac{\lambda_{t s}^{M}}{\mu_{s}^{M}}\right)^{l}}{l !}} \\
P_{b}^{m}=\frac{\frac{\left(\frac{\lambda_{t s}^{m}}{\mu_{s}^{m}}+\frac{\lambda_{t f}^{m}}{\mu_{f}^{m}}\right)^{c^{m}}}{c^{m} !}}{\sum_{l=0}^{c^{m}} \frac{\left(\frac{\lambda_{t s}^{m}}{\mu_{s}^{m}}+\frac{\lambda_{t f}^{m}}{\mu_{f}^{m}}\right)^{l}}{l !}} .
\end{gathered}
$$

We need to determine the four aggregate traffic rates $\lambda_{t f}^{M}, \lambda_{t s}^{M}, \lambda_{t s}^{m}$ and $\lambda_{t f}^{m}$ and the four service rates $\mu_{f}^{M}, \mu_{s}^{M}$, $\mu_{s}^{m}$ and $\mu_{f}^{m}$ by following the analysis model in [12] and [14], respectively. These traffics are composed of new calls, handoff calls, overflow calls, and channel-sharing calls are shown in Fig. 4, and they are all assumed to follow the Poisson process. The other parameters related to our analysis are summarized in Table 2. 


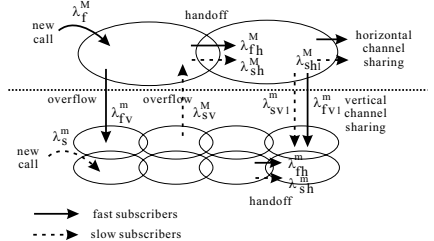

(a)

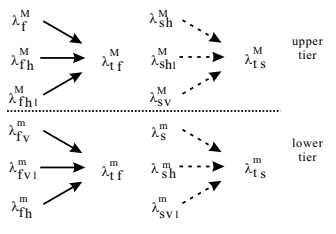

(b)
Fig. 4: (a) traffic flows for fast subscribers (solid lines) and slow subscribers (dashed lines), and (b) flow contributed to the aggregate traffic rates.

\begin{tabular}{|c|c|c|c|c|}
\hline & \multicolumn{2}{|c|}{ Macrocell } & \multicolumn{2}{c|}{ Microcell } \\
\hline Parameters & fast & Slow & fast & slow \\
\hline new call traffic rate & $\lambda_{f}^{M}$ & & & $\lambda_{s}^{m}$ \\
\hline handoff traffic rate & $\lambda_{f h}^{M}$ & $\lambda_{s h}^{M}$ & $\lambda_{f h}^{m}$ & $\lambda_{s h}^{m}$ \\
\hline overflow traffic rate & & $\lambda_{s v}^{M}$ & $\lambda_{f v}^{m}$ & \\
\hline vcs traffic rate & $\lambda_{f v l}^{M}$ & $\lambda_{s v l}^{M}$ & $\lambda_{f v l}^{m}$ & $\lambda_{s v l}^{m}$ \\
\hline hcs traffic rate & $\lambda_{f h l}^{M}$ & $\lambda_{s h l}^{M}$ & \multicolumn{2}{|l}{} \\
\hline the aggregate traffic rate & $\lambda_{t f}^{M}$ & $\lambda_{t s}^{M}$ & $\lambda_{t f}^{m}$ & $\lambda_{t s}^{m}$ \\
\hline
\end{tabular}

Table 2: Traffic parameters used in the analysis, where the vcs is vertical channel-sharing, and the hes is horizontal channel-sharing.

Variable $\lambda_{t f}^{M}$ is the aggregate traffic rate incurred by new calls, handoff calls and horizontal channel-sharing calls into a macrocell by fast subscribers:

$$
\lambda_{t f}^{M}=\lambda_{f}^{M}+\lambda_{f h}^{M}+\lambda_{f h l}^{M},
$$

where

$$
\lambda_{f h}^{M}=\lambda_{t f}^{M}\left(1-P_{b}^{M}\right) P_{f h}^{M},
$$

means the handoff rate is the aggregate traffic rate itself successfully stays in the macrocell $\left(\lambda_{t f}^{M}\left(1-P_{b}^{M}\right)\right)$ times the handoff probability $\left(P_{f h}^{M}\right)$. The last term $\lambda_{f h l}^{M}$ is caused by our horizontal channel-sharing strategy,

$$
\lambda_{f h l}^{M}=\left(\lambda_{f}^{M}+\lambda_{f h}^{M}\right) P_{b}^{M} P_{b}^{m} P_{v},
$$

which equals the new call arrival rate and handoff rate into a macrocell $\left(\lambda_{f}^{M}+\lambda_{f h}^{M}\right)$, times the probabilities that they see no free channel in the macrocell $\left(P_{b}^{M}\right)$, and neither in the microcell $\left(P_{b}^{m}\right)$, times the probability that they fail in vertical channel-sharing $\left(P_{v}\right)$. Similarly, $\lambda_{t s}^{M}$ is the aggregate traffic rate incurred by overflow calls, handoff calls and horizontal channel-sharing calls into a macrocell by slow mobile subscribers:

$$
\lambda_{t s}^{M}=\lambda_{s v}^{M}+\lambda_{s h}^{M}+\lambda_{s h l}^{M},
$$

where

$$
\lambda_{s v}^{M}=n \lambda_{t s}^{m} P_{b}^{m},
$$

means the overflow rate incurred by overflow from the $n$ microcells covered by the macrocell. The second term $\lambda_{s h}^{M}$ is the handoff calls into a macrocell by slow mobile subscirbers, which equals the slow subscribers successfully staying on the high tier $\left(\lambda_{t s}^{M}\left(1-P_{b}^{M}\right)\right)$ times the handoff probability $P_{s h}^{M}$, that is

$$
\lambda_{s h}^{M}=\left(\lambda_{t s}^{M}\right)\left(1-P_{b}^{M}\right) P_{s h}^{M} .
$$

The last term $\lambda_{s h l}^{M}$ is caused by our horizontal channelsharing strategy,

$$
\lambda_{s h l}^{M}=n\left(\lambda_{s}^{m}+\lambda_{s h}^{m}\right) P_{b}^{m} P_{b}^{M} P_{v},
$$

which equals the new call arrival rate and handoff rate of slow subscribers into the $n$ microcells $\left(n \lambda_{s}^{m}\right)$, times the probabilities that they see no free channel in the local microcell $\left(P_{b}^{m}\right)$, and neither in the macrocell $\left(P_{b}^{M}\right)$, times the probability that they fail in vertical channel-sharing $\left(P_{v}\right)$. Rate $\lambda_{t s}^{m}$ is the summation of new calls, handoff calls, and calls caused by channel-sharing for slow subscribers:

$$
\lambda_{t s}^{m}=\lambda_{s}^{m}+\lambda_{s h}^{m}+\lambda_{s v l}^{m},
$$

where $\lambda_{s h}^{m}$ is the handoff calls

$$
\lambda_{s h}^{m}=\lambda_{t s}^{m}\left(1-P_{b}^{m}\right) P_{s h}^{m},
$$

and $\lambda_{s v l}^{m}$ is caused by our vertical channel-sharing strategy

$$
\lambda_{s v l}^{m}=\frac{1}{n}\left(\lambda_{s v l}^{M}+\lambda_{f v l}^{M}\right) \frac{\lambda_{t s}^{M}}{\lambda_{t f}^{M}+\lambda_{t s}^{M}} .
$$

The summation $\lambda_{s v l}^{M}+\lambda_{f v l}^{M}$ is the overall load caused by channel-sharing (including slow and fast subscribers) in the physical area covered by a macrocell (including one macrocell and $n$ microcells), but only a fraction $1 / n$ of the load will be injected to the microcell. Rate $\lambda_{s v l}^{M}$, which counts for channel-sharing rates caused by slow subscribers, can be derived as

$$
\lambda_{s v l}^{M}=n\left(\lambda_{s}^{m}+\lambda_{s h}^{m}\right) P_{b}^{m} P_{b}^{M},
$$

Rate $\lambda_{f v l}^{M}$, which counts for channel-sharing rates caused by fast subscribers, can be derived as

$$
\lambda_{f v l}^{M}=\left(\lambda_{f}^{M}+\lambda_{f h}^{M}\right) P_{b}^{M} P_{b}^{m} .
$$

Finally, note that the last term $\frac{\lambda_{t s}^{M}}{\lambda_{t f}^{M}+\lambda_{t s}^{M}}$ is the ratio of channel-sharing flows by slow subscriber into microcells.

The last rate $\lambda_{t f}^{m}$ is the summation of handoff calls, overflow calls, and calls caused by channel-sharing for fast subscribers:

$$
\lambda_{t f}^{m}=\lambda_{f h}^{m}+\lambda_{f v}^{m}+\lambda_{f v l}^{m},
$$

where

$$
\begin{gathered}
\lambda_{f h}^{m}=\lambda_{t f}^{m}\left(1-P_{b}^{m}\right) P_{f h}^{m}, \\
\lambda_{f v}^{m}=\frac{1}{n} \lambda_{t f}^{M} P_{b}^{M}, \\
\lambda_{f v l}^{m}=\frac{1}{n}\left(\lambda_{s v l}^{M}+\lambda_{f v l}^{M}\right) \frac{\lambda_{t f}^{M}}{\lambda_{t f}^{M}+\lambda_{t s}^{M}} .
\end{gathered}
$$

The rationale is similar to the previous rate. The handoff rate $\lambda_{f h}^{m}$ is the rate for fast subscribers successfully staying in microcell $\left(\lambda_{t f}^{m}\left(1-P_{b}^{m}\right)\right)$, times the probability for them to take a handoff to neighboring microcells $\left(P_{f h}^{m}\right)$. The overflow rate $\lambda_{f v}^{m}$ for fast mobile from macrocell to microcell is $\frac{1}{n} \lambda_{t f}^{M} P_{b}^{M}$. The $\lambda_{f v l}^{m}$ is caused by our vertical channel-sharing strategy, where the ratio $\frac{\lambda_{t f}^{M}}{\lambda_{t f}^{M}+\lambda_{t s}^{M}}$ is the ratio of channel-sharing flows by fast subscriber into microcells. 


\begin{tabular}{|c|c|c|}
\hline & $P_{l f}$ & $P_{l s}$ \\
\hline TB & $P_{b}^{M} P_{b}^{m}$ & $P_{b}^{m} P_{b}^{M}$ \\
\hline CR & $P_{b}^{M} P_{R}$ & $P_{b}^{m} P_{r} P_{b}^{M}$ \\
\hline VCS & $P_{b}^{M} P_{b}^{m} P_{v}$ & $P_{b}^{m} P_{b}^{M} P_{v}$ \\
\hline VHCS & $P_{b}^{M} P_{b}^{m} P_{v} P_{R}$ & $P_{b}^{m} P_{b}^{M} P_{v} P_{r}$ \\
\hline
\end{tabular}

Table 3: Comparison of call loss probabilities for fast and slow subscribers.

\section{Performance Comparisons}

\subsection{Numerical Results}

This section compares our strategy against the take-back (TB) strategy [12] and the channel rearrangement (CR) strategy [13]. Table 3 shows the call loss probabilities for fast and slow subscribers in these strategies. VCS is to apply our vertical channel-sharing only, and VHCS to apply both our vertical and horizontal channel-sharing.

To see how these formulas compared to each other, we plug-in the following parameters. The radius of macrocells is set to $400 \mathrm{~m}$, while that of microcells $200 \mathrm{~m}$. The average velocities are $5 \mathrm{~km} / \mathrm{hr}$ and $30 \mathrm{~km} / \mathrm{hr}$ for slow and fast subscribers, respectively. The mean holding time of a call is 110 seconds. A macrocell covers $n$ microcells. The call arrival rate is $p \lambda$ for each microcell, and $n(1-p) \lambda$ for each macrocell, where $p$ is to tune the amount of fast subscribers in an area and $n$ is to take care of size difference between macrocells and microcells. The numbers of channels owned by each macrocell and microcell are 29 and 7 , respectively.

An iterative method is used to compute the call loss probabilities of the compared strategies. The results at various $\lambda$ are in Fig. 5 for fast and slow subscribers. Here we use $n=4$ and $p=0.5$. For fast subscribers, the CR scheme only redirects traffic to neighboring macrocells when a macrocell is busy. The traffic load is not released effectively, so it performs the worst, as shown in the figure. The TB scheme overflows a call to the overlaid microcell with take-back strategy at cell boundaries and thus performs better. Our VCS scheme not only overflows a call to the overlaid microcell, but also pushes other calls on the macrocell to its overlaid microcell, if necessary. Intuitively, we use multiple microcells to "absort" to load on the macrocell. So it gives much lower call loss probability. Our VHCS scheme performs even better than VCS if horizontal channel-sharing is taken.

For slow subscribers, the trend is similar on VCS and VHCS, but the CR is better than TB. This is because CR takes a channel rearrangement strategy following an overflow scheme, that makes more redirecting choices than TB between two tiers.

\subsection{Simulation Results}

To verify our performance analysis, we have also developed a simulator. The simulation environment is set up similar to our analysis model. An area with $9 \times 9$ macrocells and $18 \times 18$ microcells are simulated by wrapping

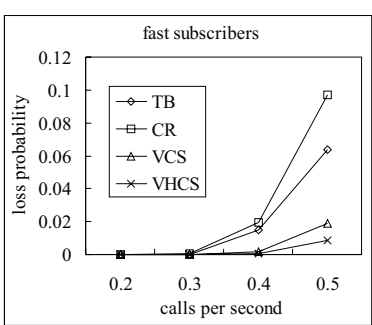

(a)

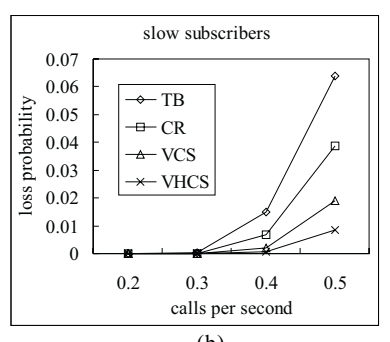

(b)
Fig. 5: Comparison based on numerical analysis on call loss probability for (a) fast subscribers and (b) slow subscribers.

around at the edges to avoid edge effects. Each macrocell covers four microcells. For simplicity, each cell is of a square shape, with macrocells being $800 \mathrm{~m} \times 800 \mathrm{~m}$ and microcells being $400 \mathrm{~m} \times 400 \mathrm{~m}$. Mobile subscribers roam in only east, west, north, and south directions. For justness with comparison on call loss probability, we follow the CR strategy [13], the subscribers never take back to their preferred tier when they have overflowed to another tier. With the TB strategy, subscribers stay on the overflowed tier, if the take-back is failed. The VCS and VHCS also follow the rules. The simulation results on call loss probability are in Fig. 6. The results are quite close to our numerical results based on analysis, which shows the correctness of our analysis.

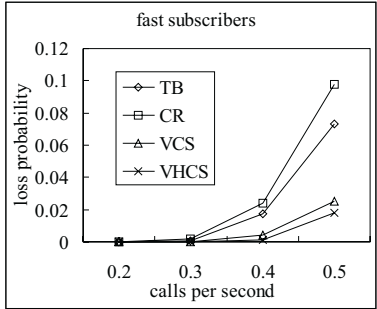

(a)

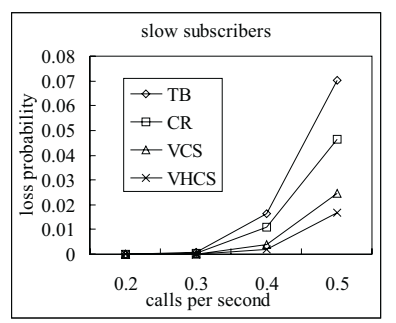

(b)
Fig. 6: Comparison based on simulation on call loss probability for (a) fast subscribers and (b) slow subscribers.

Another thing we have not observed is the call dropping probability, which is defined to be the probability that a call will be enforced to terminate because of no available channels at the events of handoffs. This is very undesirable from the users' point of view. In the call dropping simulation, all the TB, CR, VCS, and VHCS adopt the take back rules, that the target tier for handoff is always the preferred tier. As shown in Fig. 7, the trend is the same as call loss probability for both fast and slow subscribers. In fact, the gaps from CR/TB to our VCS/VHCS is increased for slow subscribers as compared to those in the comparison of call loss probabilities (refer to Fig. 6), but the gaps reduce between CR/TB and our VCS/VHCS for fast subscribers.

\section{Conclusions}

In this paper, we have proposed two channel-sharing strategies to improve the performance of a two-tier cellular system. The main idea is to share, and thus fully utilize, 


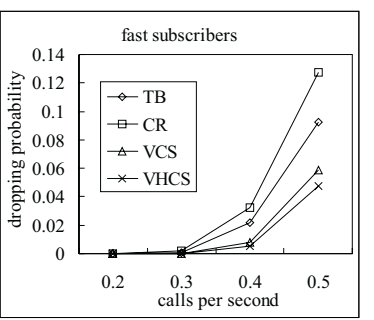

(a)

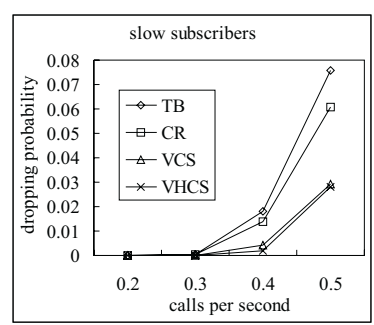

(b)
Fig. 7: Comparison based on simulation on call dropping probability for: (a) fast subscribers and (b) slow subscribers.

the channels owned by overlapping macrocells and microcells. Performance analyses based on fluid flow model and simulations are presented. Significant reduction in call loss probability and call dropping probability can be obtained over existing schemes by simply using our vertical channel-sharing strategy. Combining our horizontal channel-sharing can even slightly increase the number of calls being accepted, at the cost of slightly higher call dropping probability. As to future research, we are currently investigating the integration of our concept into a wireless ATM network.

\section{References}

[1] A. S. Anpalagan and L. Katzela. Overlaid cellular system design with cell selection criteria for mobile wireless users. In 1999 IEEE Canadian Conference on Electrical and Computer Engineering, 1999, 2428.

[2] M. Benveniste. Cell selection in two-tier microcellular/macrocellular systems. In GLOBECOM'95, 1995, 1532-1536.

[3] C. L. I, L. J. Greenstein, and R. D. Gitlin. A microcell/macrocell cellular architecture for low- and highmobility wireless users. J. on Selected Areas in Commun., 11, Aug. 1993, 885-891.

[4] D. Kim, B. W. Lim, and D. G. Jeong. An efficient paging scheme for overlaid microcell/macrocell systems. In 1996 5th IEEE International Conference on Universal Personal Communications, 1996, 961-964.

[5] Y. I. Kim, K. J. Lee, and Y. O. Chin. Effect of handoff area variation on PCS system traffic. In IEEE International Conference on Personal Wireless Communications, 1996, 134-139.

[6] K. L. Yeung and S. Nanda. Channel management in microcell/macrocell cellular radio systems. IEEE Trans. on Vehicular Technology, 45, Nov. 1996, 601612.

[7] K. L. Yeung and S. Nanda. Optimal mobiledetermined micro-macro cell selection. In VTC'95, 1995, 294-299.
[8] C. W. Sung and W. S. Wong. User speed estimation and dynamic channel allocation in hierarchical cellular system. In IEEE Veh. Technol. Cong. (VTC'94), 1994, 91-95.

[9] S. Rappaport and L. R. Hu. Microcellular Communications Systems with Hierarchical Macrocell Overlays: Traffic Performance models and analysis. In Proceeding of IEEE, 82, September 1994.

[10] X. Lagrange and P. Godlewski. Performance of a hierarchical cellular network with mobility-dependent hand-over strategies. In IEEE Veh. Technol. Cong. (VTC'96), 1996.

[11] S. A. El-Dolil, W. C. Wong, and R. Steele. Teletraffic performance of highway microcells with overlay macrocells. J. on Selected Areas in Commun., 7, January 1989.

[12] Bijan Jabbari and Woldemar F. Fuhrmann. Teletraffic Modeling and Analysis of Flexible Hierarchical Cellular Networks with Speed-Sensitive Handoff Strategy. J. on Selected Areas in Commun., 15, October 1997, 1539-1548.

[13] S. Marano, C. Mastroianni, and R. Riccardi. Performance of a micro-macro cellular system with overlapping coverage and channel rearrangement techniques. In IEEE Symposium on Computers and Communications, 1998, 705-710.

[14] B. Jabbari. Teletraffic aspects of evolving and next generation wireless communication networks. In IEEE Personal Communications, Dec. 1996, 4-9. 